\title{
LA TESIS DE LA ELECCIÓN DIVINA DE LO ÓPTIMO: UNA EXCEPCIÓN EN LA TEORÍA MODAL DE LEIBNIZ
}

\author{
MAXIMILIANO ESCOBAR VIRÉ \\ Universidad de Buenos Aires \\ Universidad Nacional de Quilmes, CONICET \\ m_escobarvire@yahoo.com
}

\section{Received:30.11.2013; Revised:17.10.2014; Accepted:16.03.2015}

Resumen: La proposición "Dios elige lo mejor" constituye una verdad incuestionable para Leibniz, y una premisa fundamental en su explicación de la existencia del mundo, tanto como en su teodicea. Leibniz sintió la necesidad de clarificar su carácter modal, dada la importancia de tal cuestión en relación con la libertad divina. Sin embargo, en el abordaje de este problema, se vio conducido a infringir los criterios de su propia teoría modal, con el fin de justificar la contingencia de tal proposición. Este trabajo intenta mostrar que la posición principal sostenida por Leibniz, en torno a la modalidad de esta proposición, constituye una suerte de excepción en el marco de su doctrina modal, y que esta ambigüedad refleja las razones profundas de las oscilaciones constatables en sus escritos sobre esta temática.

Palabras clave: Necesidad, Contingencia, Elección de lo mejor, Libertad, Dios.

\section{The Thesis of God's Choice of The Best: an Exception in The Modal Theory of Leibniz}

The proposition "God chooses the best" is for Leibniz an undeniable truth, as well as a fundamental premise in both his explanation of this world's existence and his theodicy. Leibniz thought it was necessary to clarify its modal status, in virtue of this matter's connection with God's freedom. Nevertheless, while dealing with this problem, he was led to violate his own modal theory's principles, in order to sustain the contingency of that proposition. This paper intends to show that Leibniz's main view regarding the modality of this proposition represents a sort of exception in the framework of his modal conception, and that this uncertainty reveals the profound reasons of his numerous textual hesitations on the subject.

Key words: Necessity, Contingency, Choice of the best, Freedom, God.

Manuscrito - Rev. Int. Fil., Campinas, v. 38, n.1, pp. 129-165, jan.-jun. 2015. 


\section{Introducción}

Leibniz es conocido por haber sostenido que Dios ha creado el mejor de los mundos posibles. Es curioso, sin embargo, que no haya sido igualmente famoso por haber insistido, más que cualquier otro pensador moderno, en la defensa de una creencia que constituye una de las premisas fundamentales de tal afirmación. Dicha creencia sostiene que Dios obra siempre del modo más perfecto o, también, que Dios elige siempre lo mejor.

La tesis de la elección divina de lo óptimo aparece de modo persistente en los escritos leibnizianos. Ella constituye, a mi juicio, no sólo una creencia irrenunciable del filósofo, sino también un principio investido con una importante función explicativa en su pensamiento, tanto en el plano metafísico como en el terreno moral. Esto puede verse con notable evidencia en los Ensayos de Teodicea, donde Leibniz afirma que

(...) esta suprema sabiduría [de Dios], unida a una bondad que no es menos infinita que ella, no ha podido dejar de elegir lo mejor. Porque, así como un menor mal es una especie de bien, del mismo modo un menor bien es una especie de mal, si es un obstáculo para un bien más grande. Y habría alguna cosa que corregir en las acciones de Dios, si él tuviese medio de hacer mejor. (...) si no hubiese lo mejor (optimum) entre todos los mundos posibles, Dios no habría producido ninguno." (G VI, 107)

Leibniz fue consciente de la importancia que este principio adquiría en su propia metafísica; de él dependía fuertemente el resguardo de la libertad de Dios, tanto como la defensa de su justicia ante la acusación de ser responsable por la existencia del mal. Por todo ello, sintió la necesidad de elucidar el carácter modal de esta verdad. En esta tarea, sin embargo, encontró hondas dificultades, cuyo análisis permite 
vislumbrar, a mi juicio, aspectos neurálgicos de la metafísica leibniziana, como su concepción de Dios y su comprensión de la libertad.

Este trabajo intenta exponer una de las consecuencias que se siguen del abordaje leibniziano de esta cuestión, y que pone en evidencia el carácter problemático que adquiere la posición de Leibniz en torno a la modalidad de la elección divina de lo óptimo. Tal posición conduce a admitir una suerte de excepción en el marco de su teoría modal, en la medida en que infringe sus propios criterios para el establecimiento de la distinción entre lo necesario y lo contingente. Esta excepcionalidad surge de una interpretación dual, que oscila entre dos sentidos distintos de la misma proposición. Sin embargo, tal oscilación parece arrojar luz en torno a la visión más profunda y auténtica del propio Leibniz, sobre la posibilidad de una elección divina dirigida hacia algo distinto de lo más perfecto.

\section{La distinción entre lo necesario y lo contingente en la reflexión modal de Leibniz}

Los escritos juveniles de Leibniz, en especial desde su arribo a la corte de Mainz, revelan una decidida aspiración a formular una "verdadera filosofía", capaz de conjurar el ateísmo solapado en las ideas de los "modernos" mecanicistas, a través de una metafísica concordante con las creencias fundamentales de la religión cristiana. ${ }^{1} \mathrm{Si}$ bien muchas cosas habrían de mutar en su pensamiento, la orientación a plasmar en su filosofía el acuerdo entre la razón y la fe habría de perdurar, y puede verse cristalizada en los Ensayos de Teodicea de 1710. Desde estas coordenadas iniciales de su reflexión, Leibniz se abocó a la elaboración de una metafísica teísta, en la que nada hay sin razón, y en la

\footnotetext{
${ }^{1}$ Cf. la Confessio naturae contra atheistas (A VI, iv, 489-490), y también, la carta a Thomasius de abril de 1669 (A II, i, 21-24).
}

Manuscrito - Rev. Int. Fil., Campinas, v. 38, n.1, pp. 129-165, jan.-jun. 2015. 
que un Dios personal, dotado de entendimiento y de voluntad, es la ultima ratio rerum. ${ }^{2}$ En tal metafísica, la voluntad divina constituye, ya en 1671 , la razón suficiente de la creación de un curso de eventos que es descripto como "lo mejor", y el entendimiento divino es la rąón suficiente a la que se subordina esa voluntad. ${ }^{3}$

La afirmación que sostiene que Dios quiere o elige lo mejor aparece en este marco de la explicación de la existencia del mundo, pero se encuentra íntimamente enlazada con otra aspiración leibniziana perdurable, como es la defensa de la justicia divina. Su primera formulación relevante para este contexto teórico-problemático se encuentra en el primer escrito que nos ha llegado en el que Leibniz aborda la cuestión teodiceica de la explicación sobre el origen del mal: Von der Allmacht und Allwissenheit Gottes und der Freibeit des Menschen, de 16701671.4 En ese mismo escrito, Leibniz afrontaba el problema de demostrar que la predeterminación de los acontecimientos por parte de Dios no acarrea el carácter necesario de los pecados y de la consecuente condenación de los pecadores. ${ }^{5}$ Ello revela la íntima conexión entre la cuestión modal, el problema de la libertad y la teodicea, en el marco de las líneas matrices de la metafísica leibniziana.

Leibniz parece haberse interesado primero por el análisis de las nociones modales en el contexto de sus reflexiones jurídicas. En los

2 Desde 1668-1669, Leibniz sostiene que el principio "nada es sin razón" conduce a la demostración de la existencia de Dios (A VI, i, 494). Cf. A II, i, 79. Sobre el carácter personal de Dios, asociado a la voluntad como atributo, cf. A VI, i, 301; Grua 1953, p. 118.

${ }^{3}$ Cf. A II, i, 117.

4 Cf. A VI, i, 543-544 (en adelante Von der Allmacht). La aparición más temprana de la tesis de la elección divina de lo óptimo que Grua ha detectado se encuentra en el Specimen quaestionum philosophicarum ex jure collectarum, de 1664. Cf. A VI, i, 86.

${ }^{5}$ Cf. A VI, i 538-539.

Manuscrito - Rev. Int. Fil., Campinas, v. 38, n.1, pp. 129-165, jan.-jun. 2015. 
Elementa juris naturalis, hacia 1671, emplea las definiciones de las modalidades aléticas como paradigma para esclarecer las modalidades deónticas. ${ }^{6}$ Ahora bien, como han señalado Heinrich Schepers y Hans Poser, Leibniz partía de una herencia conceptual peculiar. Esto puede verse en su clara distinción entre los conceptos de posibilidad y contingencia, que habían sido considerados como equivalentes en la tradición medieval dominante. Leibniz tomó seguramente de su maestro Thomasius una noción "moderna" del término "contingens", como opuesto subcontrario a "possibile", y como contradictorio de "necessarium".7 En otras palabras,

$>\quad$ lo posible es aquello que no es imposible, es decir, lo que puede suceder o es verdadero en algún caso;

$>\quad$ lo contingente es aquello que no es necesario, es decir, lo que puede no suceder o es falso en algún caso.

En términos más esquemáticos, las nociones modales que Leibniz ofrece en estos escritos jurídicos pueden comprenderse a través de un cuadro de oposiciones lógicas análogo al de los juicios categóricos, tal como se acostumbraba en los tratados lógicos medievales. ${ }^{8}$ Las definiciones leibnizianas corresponden a tres puntos de vista distintos: i) un punto de vista ontológico, referido a los estados de cosas, ii) un punto de vista semántico, basado en una concepción frecuencialista de la verdad, y iii) una perspectiva epistémica, basada en

\footnotetext{
${ }^{6}$ Cf. A VI, i, 398, 466 y 481; A VI, ii, 528. Sobre el empleo de esta clasificación que hace Leibniz en estos escritos, como modelo para establecer las relaciones lógicas entre las modalidades deónticas, cf. Esquisabel (2011).

${ }^{7}$ Cf. Schepers (1999, pp. 44-52, 60-63); Poser (1969, pp. 11, 14-18).

${ }^{8}$ Cf. Schepers (1999, pp. 44-46); Knuuttila (2008, pp. 531-532).

Manuscrito - Rev. Int. Fil., Campinas, v. 38, n.1, pp. 129-165, jan.-jun. 2015.
} 
la idea (de inspiración cartesiana) de una "comprensión clara y distinta": 9

\begin{tabular}{|l|l|}
\hline "Necessarium" & "Impossibile" \\
aquello que & aquello que \\
i) no puede no suceder & i) no puede suceder \\
ii) en ningún caso no es verdadero & ii) en ni un caso es verdadero \\
iii) su opuesto no se entiende clara & iii) no se entiende clara y \\
y distintamente & distintamente \\
\hline "Possibile" & "Contingens" \\
aquello que & aquello que \\
i) puede suceder & i) puede no suceder \\
ii) en algún caso es verdadero & ii) en algún caso no es verdadero \\
iii) se entiende clara y & iii) su opuesto se entiende clara y \\
distintamente & distintamente \\
\hline
\end{tabular}

Como refleja este cuadro, la noción de contingencia es empleada, por Leibniz, para caracterizar a las proposiciones verdaderas que podian haber sido falsas. Para él, como ha señalado Poser, las proposiciones contingentes son las verdades de hecho, referidas al mundo actual. En cambio, la categoría de posibilidad es empleada para referir a las series de cosas no actualizadas, pero que no se vuelven por ello imposibles. ${ }^{10} \mathrm{Si}$ bien en

\footnotetext{
${ }^{9}$ Cf. Esquisabel (2011, pp. 8-9).

${ }^{10}$ Cf. Poser (1969, pp. 30-33). Poser distingue entre verae contingentes y falsae contingentes, a las que Leibniz menciona en las Generales inquisitiones. El problema que puede suscitar el soslayar esta clarificación del uso leibniziano de los términos, es el que se refiere a la consideración de las proposiciones sobre mundos posibles no actualizados como verdades contingentes, tal como lo
} 
algunos pasajes Leibniz reconoce que las cosas posibles no-necesarias son, en general, contingentes (así como las cosas necesarias son, de igual modo, posibles), ${ }^{11}$ para él, lo contingente es ante todo lo actual, aquello que es pero que pudo haber sido de otro modo.

El interés leibniziano por la teoría modal, sin embargo, pronto se enlaza a la temática metafísica general de la fundamentación de la libertad y la defensa de la justicia divina, mucho más acuciante para sus aspiraciones filosóficas. Así puede verse en Von der Allmacht, donde Leibniz ya emplea los conceptos modales como vía para escapar a una visión necesitarista, y anuncia su posterior apropiación de la distinción entre necesidad absoluta y necesidad hipotética. ${ }^{12}$ Hacia 1672-1673, en su Confessio philosophi, la definición de los modalizadores aparece como clave en la estrategia argumental para rechazar el carácter necesario de todos los eventos. Desde entonces, la reflexión modal tendría para Leibniz una relevancia metafísica fundamental, dado que la

sostienen Adams y Hacking. Cf. Adams (1994, pp. 30-34); Hacking (1982, p. 190).

${ }^{11}$ Cf. G VI, 441.

12 Cf. A VI, i, 539-541. Leibniz se opone al sophisma según el cual mi propia condenación es necesaria, en razón de haber sido prevista por Dios. Para fundamentar esta oposición, la reflexión teológico-modal medieval aportaba una distinción conceptual crucial: aquella entre una necesidad en sentido primario o absoluta, y una necesidad derivada o bipotética. La diferencia residía en que esta última constituía una necesidad condicional, resultante de una bipótesis, mientras que la primera constituía una necesidad incondicionada, fundada en la naturaleza de las cosas. En tal sentido, una concepción necesitarista sería, para Leibniz, aquella en la cual todo cuanto sucede es necesario en sentido primario o absoluto, es decir, por sí mismo y no como consecuencia de una condición (como sería la previsión divina). Bajo tal concepción, no existiría ninguna posibilidad, ni siquiera contrafáctica, de que las cosas hubieran ocurrido de otro modo. Para Leibniz, afirmar la necesidad absoluta de todas las cosas equivale a identificar lo actual con lo posible, y a negar toda posibilidad de eventos contrarios. Esto puede verse en su oposición al necesitarismo spinoziano. Cf. A VI, iv, 1385-1386, 1775-1776.

Manuscrito - Rev. Int. Fil., Campinas, v. 38, n.1, pp. 129-165, jan.-jun. 2015. 
preservación de la contingencia y la distinción entre lo necesario y lo contingente resultaban condición necesaria para preservar aspectos irrenunciables a una cosmovisión cristiana, como la libertad y la responsabilidad moral, tanto en las creaturas como en Dios.

En la Confessio philosophi, sin embargo, Leibniz da un paso más en su reflexión modal. En sus escritos jurídicos se había centrado en el esclarecimiento de las relaciones lógico-formales entre los modalizadores, es decir, en una lógica modal. El diálogo de 1672-1673 aporta el esbozo de una metafísica modal, ya que propone una condición metafísica como fundamento de las categorías modales. Tal condición se encuentra en las "esencias" o "ideas" de las cosas, y se explica en términos lógicos: posible es aquello cuya idea o esencia no implica contradicción, por lo cual se puede entender clara y distintamente. En tal sentido, algo será imposible si su propia esencia o idea es contradictoria. ${ }^{13}$ Por eso Leibniz se refiere a estas nociones en términos de lo que es posible o imposible "per se", esto es, por sí mismo. ${ }^{14}$

Esta visión puede caracterizarse como la teoría leibniziana de las modalidades per se. ${ }^{15}$ Dicha teoría distingue entre una necesidad absoluta, aquella cuyo contrario implica contradicción en su propia idea o esencia, y una necesidad hipotética, que es la necesidad de aquello que es consecuencia de otra cosa (una bipótesis), pero cuyo contrario es posible per

13 Cf. A VI, iii, 127-128, donde Leibniz se refiere tanto a la esencia como a la idea de las cosas.

14 En su escrito titulado Principium meum, est quicquid existere potest et aliis compatibile est, id existere, del 2 de diciembre de 1676, Leibniz habla de las cosas que son posibles per se ("possibilia per se", cf. A VI, iii, 581). Asimismo, en sus notas sobre la conversación con Niels Stensen de 1677, Leibniz distingue una necesidad per se de una necesidad hipotética o per accidens, y habla también de una imposibilidad hipotética (cf. A VI, iv, 1377-1378).

15 Esta denominación sigue la terminología empleada por Robert Sleigh. Cf. S1 xxv-xxvi.

Manuscrito - Rev. Int. Fil., Campinas, v. 38, n.1, pp. 129-165, jan.-jun. 2015. 
se. Leibniz identifica, en ocasiones, a la primera con la necesidad del consecuente, y a la segunda con la necesidad de la consecuencia, según otra distinción tradicional. ${ }^{16}$ En mi interpretación, las "esencias" o "ideas" en las que Leibniz funda las nociones modales primarias o absolutas deben ser identificadas con las ideas de las cosas en la mente de Dios, ya que así lo requiere su metafísica, y así lo sugiere él mismo en algunos escritos de la década de $1670 .{ }^{17}$

Sin embargo, como se sabe, Leibniz reformuló esta concepción modal, y pasó a adoptar, en 1686, lo que ulteriormente habría de considerar como su explicación definitiva de la diferencia entre las proposiciones necesarias y las contingentes. Esa explicación afirmaba que el rasgo distintivo de las proposiciones existenciales o verdades de becho residía en que, en ellas, el análisis requerido para demostrarlas era infinito, por lo cual resultaban indemostrables en un número finito de pasos. ${ }^{18}$ Las proposiciones necesarias, por el contrario, eran demostrables en un análisis finito. Esta diferencia modal, centrada ahora en el tipo de prueba, resultaba vital para salvar la distinción entre lo necesario y lo contingente, dado que Leibniz, desde 1679, había pasado a definir la naturaleza de la verdad en términos de la inclusión conceptual del predicado en el sujeto. Tal diferencia permitía, también,

16 Cf. A VI, iv, 1652; G III, 400; G VI, 380. La necessitas consequentiae recaía sobre un condicional ("A entonces B" es necesario), mientras que la necessitas consequentis, a la que Leibniz asocia con la necesidad absoluta, recaía sobre el consecuente de un condicional (si "A" es verdadero, entonces "B" es necesario). Cf. Adams (1994, p. 16).

17 Algunos manuscritos de 1677, en los que Leibniz infiere la existencia de Dios por la realidad de las verdades eternas, muestran que identifica las "esencias" o "naturalezas" en las que se originan tales verdades con las ideas que subsisten en Dios, a quien califica como el "sujeto de las ideas". Cf. A VI, iv, 17-19.

${ }^{18}$ En especial a partir de las Generales Inquisitiones. Cf. A VI, iv, 758, 763 y 776777. Cf. también A VI, iv, 1515-1516.

Manuscrito - Rev. Int. Fil., Campinas, v. 38, n.1, pp. 129-165, jan.-jun. 2015. 
caracterizar el concepto de sustancia como ens completum, cuya noción individual incluye la totalidad de sus predicados verdaderos. ${ }^{19}$

Si bien la explicación basada en el análisis infinito parecía reservar los modalizadores para las proposiciones, Leibniz nunca fue muy preciso en su empleo de las nociones modales, lo cual dificulta la tarea de su clarificación. Para él, tales nociones se predican indistintamente sobre proposiciones, eventos, entes (como el Ens necessarium) y, también, sobre las elecciones efectivas de los espíritus. ${ }^{20}$ Sin embargo, para todos estos modalizanda, Leibniz siempre aceptó un mismo criterio modal general, según el cual la distinción entre lo necesario y lo contingente implica, por definición, una distinción entre aquello cuyo contrario es o no es posible, ya sea que se conciba esta posibilidad en términos de la consistencia lógica interna de la noción considerada, o bien en términos de la indemostrabilidad de la proposición por medio de un análisis finito. En este sentido, Leibniz escribe, en un pasaje de 1705: "necesario es aquello que es lo único posible". ${ }^{21}$ Asimismo, en 1706, afirma que no reconoce como necesaria ninguna proposición "que no pueda ser demostrada por una reducción a aquello de lo cual lo contrario implica contradicción". 22 Y en los Ensayos de Teodicea, declara que la necesidad y la posibilidad, "tomadas metafísicamente y en rigor", dependen de una única cuestión: "si el objeto en sí mismo o aquello que le es opuesto implica contradicción o no". 23

Así pues, desde las coordenadas de la reflexión modal leibniziana, el esclarecimiento del carácter modal de la proposición "Dios elige lo mejor" acarrea claras implicancias en relación a la

\footnotetext{
${ }^{19}$ Cf. A VI, iv, 400, 559.

${ }^{20}$ Cf. por ejemplo A VI, iii, 127-128.

$21 \mathrm{Gr} 475$.

22 Cf. Gr 493.

23 G VI, 333.
}

Manuscrito - Rev. Int. Fil., Campinas, v. 38, n.1, pp. 129-165, jan.-jun. 2015. 
posibilidad de la proposición contraria. Tales implicancias constituirán una prueba difícil para el abordaje leibniziano.

\section{La posición leibniziana en torno al carácter modal de la elección divina de lo óptimo}

Desde 1671, en escritos como Von der Allmacht y la carta a Magnus Wedderkopf, Leibniz se había referido a la elección divina de lo óptimo en términos que sugieren fuertemente una consideración de la misma como necesaria. Allí afirmaba que resulta "imposible", para Dios, no ser "necesitado hacia lo mejor por la misma idealidad de las cosas", ${ }^{24}$ y que negar esta voluntad de lo óptimo en un ser omnisciente implica trastocar los términos. ${ }^{25}$ Asimismo, en la Confessio philosophi, afirmaba que "la libertad de Dios es suprema aunque no pueda errar en la elección de lo mejor". ${ }^{26}$ En tales escritos, Leibniz no se planteaba expresamente aún el problema de esclarecer la modalidad correspondiente a la elección en cuestión, pero sí reconocía la necesidad de conciliar el carácter cierto e infalible de esa elección con la libertad de Dios.

En este sentido, la posición leibniziana en torno a la naturaleza de la libertad, durante la década de 1670, permanecería fiel a una orientación claramente intelectualista. De acuerdo al análisis de Michael Murray, el enfoque intelectualista, en el siglo XVII, se oponía a la doctrina de la indiferencia de equilibrio, afirmando que la determinación de la voluntad, en toda elección, procede del juicio del entendimiento sobre lo mejor, y no de una operación de la voluntad, indiferente

\footnotetext{
${ }^{24}$ Cf. A II, i, 117.

25 Cf. A VI, i, 543-544.

26 A VI, iii, 135.
}

Manuscrito - Rev. Int. Fil., Campinas, v. 38, n.1, pp. 129-165, jan.-jun. 2015. 
respecto de la consideración intelectual del bien. ${ }^{27}$ Tal rechazo se encuentra expresamente en Leibniz, ${ }^{28}$ quien hace coincidir, en Dios, la "suprema libertad" con el hecho de "ser forzado" hacia lo mejor por la razón. ${ }^{29}$ Esta coincidencia es la que se volverá problemática a sus ojos.

Leibniz comienza a tematizar el carácter modal de la elección divina de lo óptimo a partir de algunos escritos de 1677.30 En ellos, el interés explícito reside en reafirmar la compatibilidad entre la libertad de Dios y la necesaria tendencia de su voluntad hacia lo mejor, de acuerdo con sus ideas de los años previos. Pero esa primera tematización se torna en problematización recién con los manuscritos de 1680-1686.31 Aquí también, la preocupación leibniziana se centra en la libertad divina. Entre estos dos momentos puede notarse un cambio importante. En el primer abordaje de 1677, la posición leibniziana era favorable al carácter necesario de la elección en cuestión, si bien, lamentablemente, Leibniz nunca aclara el tipo de necesidad que concede

${ }^{27}$ Cf. Murray (1996, pp. 31-33). Murray se refiere a la visión que adhería a la indiferencia de equilibrio como "voluntarismo", empleando una terminología habitual en el análisis de las doctrinas de la libertad en los siglos XVI y XVII. $\mathrm{Su}$ posición es que Leibniz rechazó tanto el intelectualismo como el voluntarismo, y adhirió a lo que él denomina como el "necesitarismo moral" (op. cit., p. 27). Mi interpretación es que Leibniz se desplaza de una posición inicialmente más intelectualista hacia una visión más cercana a este necesitarismo moral, en especial a partir de su incorporación del discurso de las "razones inclinantes".

${ }^{28}$ Cf. por ejemplo A VI, i, 544-545.

${ }^{29}$ Cf. A II, i, 117-118; A VI, iii, 135.

${ }^{30}$ Esto puede verse en un breve escrito titulado De necessitate eligendi optimum (cf. A VI, iv, 1351-1352), pero sobre todo en las notas de la conversación con Stensen de ese mismo año (cf. A VI, iv, 1383).

31 Principalmente en los textos titulados De libertate et necessitate (cf. A VI, iv, 1445-1447), De necessitate et contingentia (cf. A VI, iv, 1449) y De libertate a necessitate in eligendo (cf. A VI, iv, 1453, 1454-1455). También en el importante escrito titulado De libertate, fato, gratia Dei (cf. A VI, iv, 1598-1601) y en las notas sobre Bellarmino (cf. A VI, iv, 2577).

Manuscrito - Rev. Int. Fil., Campinas, v. 38, n.1, pp. 129-165, jan.-jun. 2015. 
para ella. Sin embargo, esta posición evidencia un giro abrupto hacia 1680, época en que Leibniz pasa a sostener decididamente la contingencia de la proposición "Deus vult eligere perfectissimum".32

La proposición en cuestión presenta dificultades interpretativas, como se verá. Una primera dificultad reside en esclarecer si la modalidad (necesidad o contingencia) recae sobre el acto de elección mismo, o bien sobre el hecho de que Dios elija lo mejor toda vez que elige algo, siendo el acto de elección resultado de una resolución libre. En el primer caso, la modalidad recaería sobre la proposición "Dios elige lo mejor", y establecería la necesidad o contingencia del hecho de que Dios elija. En cambio, en la segunda interpretación, la modalidad recaería sobre la proposición "Si Dios elige, elige lo mejor". En este caso, se asume que es contingente el que Dios elija, ya que elije libremente; lo que sería necesario o contingente es que, cuando elige, elija lo óptimo.

La interpretación más adecuada es, seguramente, la segunda. Esto se debe a que, para Leibniz, Dios no estaba necesitado a crear un mundo posible, sino que podía no haber creado nada, si no hubiese encontrado un mundo que fuese el mejor. En este sentido, la tesis de la elección divina de lo óptimo $(T)$ puede reformularse, a los fines de su comprensión, como una proposición condicional cuantificada universalmente:

T: Para todo aquello que Dios puede elegir, si Dios elige, entonces elige lo mejor ${ }^{3}$

\footnotetext{
32 Un escrito de 1678-1681, titulado Deus nibil vult sine ratione, parece anticipar este giro. Cf. A VI, iv, 1389.

33 Leibniz siempre formula esta tesis como una proposición categórica, no condicional, por lo que $T$ debe verse como una reconstrucción analítica que,

Manuscrito - Rev. Int. Fil., Campinas, v. 38, n.1, pp. 129-165, jan.-jun. 2015.
} 
Que sea dada una situación de elección depende, en la metafísica leibniziana, de que sea concebible, para el entendimiento divino, una pluralidad de cosas posibles per se pero incomposibles entre sí. Leibniz afirma constantemente que no habría ninguna elección, si no hubiese alternativas posibles. ${ }^{34}$ Ahora bien, dado que existen infinitas series posibles in mente Dei, la voluntad divina queda enfrentada a la posibilidad de elegir o no elegir, y también, eventualmente, a la determinación de cuál serie elegir. Según creo, el principio de la elección de lo óptimo rige desde el principio todas estas alternativas, y por ello es crucial esclarecer su modalidad.

La relación de Leibniz con la elección divina de lo óptimo fue un tanto oscilante, como bien ha notado Adams. ${ }^{35}$ Pueden encontrarse varios pasajes, posteriores a 1690, en los que afirma o sugiere claramente que la proposición es más bien necesaria. ${ }^{36}$ Así, por ejemplo, en unas notas redactadas en 1698, Leibniz afirma:

Hablando en términos absolutos, debe decirse que otro estado [del mundo] puede existir, pero sin embargo existe el presente, porque a partir de la naturaleza de Dios se sigue que prefiera lo más perfecto. (Gr 393)

En otros escritos de la misma época, sin embargo, ratifica el carácter contingente de la elección divina. Tal es la posición consagrada en los Nuevos ensayos sobre el entendimiento bumano. ${ }^{37}$

sin embargo, intenta expresar con mayor claridad el significado de la afirmación leibniziana.

${ }^{34}$ Cf. por ejemplo G VI, 258, 441.

35 Cf. Adams (1994, p. 36).

${ }^{36}$ Cf. Gr 343, 360.

${ }^{37}$ Cf. A VI, vi, 178-179, 198-199.

Manuscrito - Rev. Int. Fil., Campinas, v. 38, n.1, pp. 129-165, jan.-jun. 2015. 
Conciliar estas divergencias no parece ser una tarea auspiciosa. Hay, ciertamente, una tensión persistente en los escritos leibnizianos en torno a esta cuestión. Sin embargo, en mi interpretación, hay una posición que Leibniz tendió a favorecer y a considerar como más consistente con su propia metafísica, desde 1680 y en toda su madurez. Tal posición es la que afirma la contingencia de la elección divina de lo óptimo. Dos argumentos pueden ofrecerse en defensa de esta interpretación. En primer lugar, la contingencia de la elección divina es la única posición consistente con su concepción de la voluntad y de la libertad sostenida desde principios de la década de 1680, que es aquella que se basa las "razones inclinantes". En segundo lugar, un índice importante a favor de esta lectura es que, en los últimos diez años de su vida, las oscilaciones prácticamente desaparecen, y se consolida en su discurso la atribución de una necesidad moral a dicha elección. Me referiré brevemente a estos dos argumentos.

1- La razón principal que movió a Leibniz a abordar el problema del carácter modal de esta verdad fue la necesidad que sintió de resguardar la libertad de Dios. En los manuscritos de comienzos de la década de 1680, Leibniz afirma que la proposición "Dios elige lo mejor" constituye "la primera de las proposiciones de hecho, o el origen de toda existencia contingente". Por tanto, dice, si esa proposición fuese demostrable (es decir, necesaria), Dios no habría sido libre al realizar tal elección. ${ }^{38}$ No es casual que, en esos escritos, Leibniz comience a sostener una concepción más atenuada de la libertad, incorporando la tesis según la cual las razones inclinan sin necesitar. La apelación a las razones inclinantes es invocada, en estos escritos, justamente para garantizar que Dios no es necesitado a elegir lo mejor. ${ }^{39}$ En mi interpretación, Leibniz pasa de una visión más intelectualista, que

\footnotetext{
38 Cf. A VI, iv, 1454.

${ }^{39}$ Cf. A VI, iv, 1452, 1593.
}

Manuscrito - Rev. Int. Fil., Campinas, v. 38, n.1, pp. 129-165, jan.-jun. 2015. 
admitía la compatibilidad entre la libertad y la necesidad de elegir lo mejor, a una concepción más atenuada y más inspirada en el necesitarismo moral, centrada en las "razones inclinantes", justamente a causa de la insatisfacción que terminó sintiendo frente a aquella primera admisión de una elección divina "necesitada". En su terminología, Dios ya no será "necesitado", sino "determinado" a elegir lo mejor. ${ }^{40}$

2- La necesidad moral era, para los autores jesuitas que acuñaron esta expresión, la modalidad que regía las operaciones de la voluntad, caracterizando la relación entre una elección y sus motivos. ${ }^{41} \mathrm{El}$ modelo adoptado para explicar esa relación era el de la inclinación pasional. ${ }^{42} \mathrm{La}$ apropiación leibniziana de esta noción plantea arduos desafíos interpretativos, dado que Leibniz nunca formula una clarificación precisa del sentido en que la emplea. En especial, cabe la posibilidad de que Leibniz pretenda emplear este concepto con una significación enteramente deóntica, para hacer alusión a una obligación o un deber moral. ${ }^{43}$ La elucidación de esta problemática reviste enorme importancia para la comprensión de su metafísica madura. A mi juicio, el empleo de esta noción, en los escritos leibnizianos del último decenio, tiene implicancias de orden alético. Leibniz deja en claro que la necesidad moral admite la posibilidad de lo contrario. ${ }^{44}$ Para él, dicha necesidad cualifica ante todo a la elección divina de lo óptimo, ya que se define como la necesidad con la cual el sabio elige lo mejor. Ahora bien, Leibniz la explica apelando a su teoría de las razones inclinantes. En toda elección, las opciones inclinan a la voluntad de acuerdo a la

\footnotetext{
${ }^{40}$ Cf., por ejemplo, A VI, vi, 198-199.

${ }^{41}$ Cf. Anfray (2011, pp. 68-70). Según Murray (1996, p. 38; 2005, p. 203), los teólogos jesuitas que adoptaron la expresión "necessitas moralis", en el siglo XVI, fueron Diego Montoya y Diego Ruiz de Granados.

${ }^{42}$ Cf. Anfray (2011, p. 68).

${ }^{43}$ Cf. Así lo interpreta Rateau (2008, p. 519).

${ }^{44}$ Cf. G VII, 389; G VI, 257-258.
}

Manuscrito - Rev. Int. Fil., Campinas, v. 38, n.1, pp. 129-165, jan.-jun. 2015. 
percepción de su grado comparativo de perfección. ${ }^{45}$ Así, para el Leibniz de madurez, la necesidad moral permite hallar un término medio entre dos alternativas perniciosas para la piedad: la de una necesidad ciega y la de una indiferencia de equilibrio. ${ }^{46}$

\section{El problema de la posición de Leibniz: la proposición "Dios elige lo mejor" como excepción en su teoría modal}

La justificación de la posición principal sostenida por Leibniz, favorable a la contingencia de la elección divina de lo óptimo, da lugar a un problema serio, cuyo análisis, a mi juicio, permite vislumbrar las razones profundas de la tensión que recorre sus escritos en torno a esta cuestión. En lo que sigue, intentaré formular este problema.

La contingencia de la proposición "Dios elige lo mejor", desde los parámetros modales leibnizianos, exige que se admita la posibilidad de lo contrario. Ahora bien, en su análisis, Leibniz construye de dos modos distintos la proposición contraria. Ello revela que, aun asumiendo que el acto de elección es libre, Leibniz admite dos interpretaciones distintas de la tesis. En cada una de estas lecturas, el modalizador recae sobre un elemento distinto de la proposición.

En unas notas sobre Bellarmino, de 1680-1684, Leibniz escribe:

De la esencia de Dios o de su perfección suprema se sigue de modo cierto y, si así se quiere decir, como consecuencia necesaria, que Dios elige lo mejor; pero elige lo óptimo libremente, porque en lo óptimo mismo no hay ninguna necesidad absoluta. De otro modo, su contrario implicaría contradicción, y sólo lo óptimo sería posible, mientras que las demás cosas serían imposibles, contra la hipótesis. (A VI, iv, 2577)

\footnotetext{
45 Cf. G VI, 126-127.

46 Cf. G VI, 128, 255, 323.

Manuscrito - Rev. Int. Fil., Campinas, v. 38, n.1, pp. 129-165, jan.-jun. 2015.
} 
En este pasaje, y en otros similares, ${ }^{47}$ lo contrario a la elección libre (contingente) sería que el objeto de elección fuera necesario, por lo cual sería el único posible. En esta interpretación, pues, el modalizador de necesidad parece recaer sobre el objeto de la elección. En tal sentido, la elección divina sólo podría ser necesaria si hubiese una única opción posible.

Esto es problemático, ya que ninguna elección sería posible si no hubiese opciones alternativas. Leibniz lo sabe. En parte por ello, hacia 1689, reelabora esta posición, y pasa a sostener que la elección no es necesaria porque no es necesario que el objeto que Dios elije sea el mejor:

(...) aunque se conceda que es necesario que Dios elija lo mejor, o que lo mejor sea necesario, no obstante, no se sigue que aquello que es elegido sea necesario, porque no se da ninguna demostración acerca de qué es lo óptimo. Y aquí, de algún modo, tiene lugar la distinción entre necesidad de la consecuencia y del consecuente. De modo que aquello, en definitiva, es necesario por necesidad de la consecuencia, y no del consecuente, porque es necesario en razón de que lo óptimo es supuesto, admitida la hipótesis de la infalible elección divina de lo mejor. (A VI, iv, 1652) ${ }^{48}$

Siguiendo esta interpretación de la proposición contraria, la tesis $T$ podría reformularse del siguiente modo:

$T_{i:}$ Para todo lo que Dios puede elegir, si Dios elige, entonces [M] aquello que elige es lo mejor

${ }^{47}$ Cf. por ejemplo A VI, iv, 1446-1447.

48 Cf. también Gr 336.

Manuscrito - Rev. Int. Fil., Campinas, v. 38, n.1, pp. 129-165, jan.-jun. 2015. 
En esta reformulación, el modalizador (indicado con el símbolo $[M]$ ) afecta al consecuente del condicional. Esto concuerda con lo que Leibniz afirma en estos pasajes, cuando le asigna a $T$ una necesidad hipotética.

Sin embargo, en otro escrito de 1680-1684, titulado De libertate a necessitate in eligendo, Leibniz propone una interpretación distinta:

Esta proposición ["Dios quiere lo más perfecto"] es el origen del tránsito de la posibilidad a la existencia de las creaturas. Pero preguntarás si lo contrario implica contradicción, esto es, que Dios elija aquello que no es lo más perfecto. Digo que no implica contradicción, sino una vez puesta la voluntad de Dios. (A VI, iv, 1454)

En este pasaje, la proposición contraria no refiere a la necesidad del objeto o de su carácter óptimo, sino que refiere a la posibilidad de una elección divina de lo menos perfecto. En efecto, aquí lo contrario a $T$ sería "que Dios elija aquello que no es lo más perfecto", aceptando, sin más, la posibilidad de lo menos perfecto. Por eso, en este escrito, la estrategia que adopta Leibniz para justificar la contingencia de $T$ consiste en sostener que esta última proposición ("Dios no elige lo mejor") no implica contradicción (por lo cual es posible). En tal sentido, el modalizador recae, más bien, sobre la elección, sobre el modo en que elige la voluntad divina. Aquí sí está en juego determinar si Dios quiere lo óptimo necesariamente o libremente.

Bajo esta interpretación de la tesis contraria, $T$ puede reformularse como sigue:

$T_{i i:}$ Para todo lo que Dios puede elegir, si Dios elige, entonces [M] elige lo mejor en la escala jerárquica de los bienes posibles

Manuscrito - Rev. Int. Fil., Campinas, v. 38, n.1, pp. 129-165, jan.-jun. 2015. 
Esta comprensión de la tesis coincide con numerosos pasajes, en los que Leibniz señala que Dios quiere lo óptimo porque quiere en razón de lo mejor. ${ }^{49}$

Si bien ambas interpretaciones pueden encontrarse en sus escritos, solamente en unas notas sobre Bayle, de 1706, Leibniz intenta trazar la distinción entre ellas, estableciendo dos sentidos diferenciados de la proposición. ${ }^{50}$ En la mayoría de los escritos en que defiende su posición principal, por el contrario, trata a $T$ como una proposición unívoca, y privilegia la primera interpretación $\left(T_{i}\right)$. En efecto, la estrategia argumental más habitual de Leibniz, para justificar la contingencia de $T$, consiste en sostener que lo contrario a lo que Dios elige sigue siendo posible, lo cual resguarda la posibilidad contrafáctica de una elección divina diferente. ${ }^{51}$ En su versión más elaborada (aunque menos frecuente), esta estrategia funda la contingencia de $T$ en la indemostrabilidad referida a cuál objeto es el mejor.

A mi juicio, esta estrategia es incorrecta e inadmisible. Como bien señala Paul Rateau, esta línea argumental comete un paralogismo, en tanto pretende inferir la contingencia de la elección a partir de la contingencia del objeto de elección. Según este autor, la posibilidad del objeto (contrario) a lo sumo autoriza a hablar de una elección, pero de ningún modo implica la contingencia de esa elección. ${ }^{52}$ Que el objeto elegido sea el mejor de modo contingente tampoco resuelve la cuestión. Lo que hace falta, por tanto, es demostrar que $T$ es contingente bajo la segunda interpretación, referida a la elección. Y ello equivale a demostrar

${ }^{49}$ Cf. A VI, iii, 134-135; A VI, iv, 1660-1661, 1663; G III, 402.

${ }^{50}$ Cf. Gr 493-494. La importancia de trazar esta distinción ha sido señalada por Mondadori (1986, p. 194).

${ }^{51}$ Cf. A VI, iv, 1446-1447; G VI, 128, 255, 441; G IV, 438-439; G III, 32, 402; G VII, 390 .

52 Cf. Rateau (2008, pp. 528-529).

Manuscrito - Rev. Int. Fil., Campinas, v. 38, n.1, pp. 129-165, jan.-jun. 2015. 
que es posible, para el Dios leibniziano, querer y elegir aquello que conscientemente juzga como menos perfecto.

Aquí es donde surge el problema que quiero plantear. A mi juicio, los escritos de Leibniz revelan que, para él, esta posibilidad de la proposición contraria a $T$, en tanto referida a la elección $\left(T_{i i}\right)$, resultaba inadmisible.

En un pasaje de los Ensayos de Teodicea, Leibniz ciertamente afirma que Dios podía elegir lo que no era mejor "metafísicamente hablando", aunque no "moralmente hablando". ${ }^{33}$ Sin embargo, no ofrece ningún argumento que refiera esta posibilidad a la elección. Por el contrario, a lo largo de dicha obra, la estrategia persistente es la que afirma la posibilidad del objeto contrario. Solamente en un escrito, el ya mencionado De libertate a necessitate in eligendo (de 1680-1684), Leibniz intenta formular un argumento para defender la posibilidad de una elección divina de lo menos perfecto. Se trata del sorprendente argumento en el que Leibniz postula un regreso infinito en las elecciones de Dios. ${ }^{54}$ Según dice allí, "Dios quiere querer elegir lo más perfecto, y quiere la voluntad de querer. Y así hasta el infinito". ${ }^{5}$ Sin embargo, hasta donde sabemos, Leibniz nunca más insistió con esta estrategia argumental, ya que se oponía tajantemente a su concepción de la voluntad. ${ }^{56}$

\footnotetext{
${ }^{53}$ Cf. G VI, 256.

${ }^{54}$ Cf. A VI, iv, 1454-1455.

55 A VI, iv, 1454.

56 Tal concepción era expresamente contraria a todo regreso infinito en las operaciones de la voluntad, ya que ello privaría a la elección de toda razón suficiente e implicaría una indiferencia de equilibrio. Cf. A VI, iv, 1408, el escrito Du franc arbitre, de 1678-1681.
}

Manuscrito - Rev. Int. Fil., Campinas, v. 38, n.1, pp. 129-165, jan.-jun. 2015. 
Algunos pasajes de madurez revelan claramente que Leibniz no estaba dispuesto a admitir la posibilidad de la proposición "Dios no elige lo mejor". Así, en el Compendio a los Ensayos de Teodicea, Leibniz afirma:

Si la voluntad de Dios no tuviera como regla el principio de lo mejor, se dirigiría al mal, que sería lo peor; o bien sería indiferente de algún modo al bien y al mal y estaría guiada por el azar: pero una voluntad que siempre se dejara ir al azar no valdría más, para el gobierno del universo, que el concurso fortuito de los corpúsculos exento de toda divinidad. (de Olaso 529 / G VI, 386-387)

Y en la carta a Coste del 19 de diciembre de 1707, sostiene:

Sin embargo, yo veo que hay personas que se imaginan que uno se determina a veces por la opción menos valorada, y que Dios elige a veces el menor bien, todo considerado, y que el hombre elige a veces sin motivo y contra todas sus razones, disposiciones y pasiones; en fin, que uno elige algunas veces sin que haya razón alguna que determine la elección. Pero es esto lo que yo tengo por falso y absurdo, puesto que uno de los grandes principios del buen sentido es este, que nada sucede jamás sin causa o razón determinante. Así, cuando Dios elige, es por la razón de lo mejor; cuando el hombre elige, aquello será la opción que más lo haya afectado. (G III, 402)

Estos pasajes revelan dos razones fundamentales por las cuales Leibniz rechaza la posibilidad de una elección divina de lo menos perfecto. En primer lugar, tal posibilidad se opone a un elemento irrenunciable en su concepción de la voluntad y de la libertad. Leibniz siempre sostuvo la estricta subordinación de toda elección a la consideración intelectual de lo mejor. Para él, el querer sólo es comprensible en relación al bien. Por tanto, nadie puede querer aquello que considera malo o peor. Esto vale incluso para la Medea de Ovidio,

Manuscrito - Rev. Int. Fil., Campinas, v. 38, n.1, pp. 129-165, jan.-jun. 2015. 
que quiere el mal porque el placer de la venganza prevalece, como un bien mayor, frente a la conciencia por la injusticia cometida. ${ }^{57}$ En los Ensayos de Teodicea, Leibniz recuerda el axioma según el cual "minus bonum habet rationem mall". ${ }^{58}$ No se puede querer "sub ratione mall". ${ }^{59}$ A lo sumo, la mente tiene la capacidad de suspender el juicio, al ser apartada por otros pensamientos, interrumpiendo con ello el proceso de la deliberación hasta una mejor consideración ulterior. Sin embargo, la voluntad nunca puede querer sino aquello que el entendimiento haya juzgado como lo mejor, cuando la deliberación llegue a su término. ${ }^{60}$

En segundo lugar, una elección divina de lo menos perfecto sería contraria al principio de razón suficiente. En efecto, si solamente el bien cuenta como una razón para el querer, toda elección dirigida a algo distinto de lo óptimo aparece como desprovista de razón suficiente. Por ello, no es casual que, en estos fragmentos, la posibilidad de una elección de lo peor aparezca asociada a la indiferencia de equilibrio. Tal indiferencia, para Leibniz, priva a toda elección tanto de causa eficiente como de causa final, por lo cual, en definitiva, la despoja de toda razón..$^{61}$

Una tercera razón surge de la perfección absoluta de la esencia divina. Según Leibniz, Dios es el sujeto de todas las perfecciones, y la

\footnotetext{
${ }^{57}$ Cf. A VI, iii, 134-135.

58 Cf. G VI, 231.

${ }^{59}$ Cf. A VI, iv, 1360.

${ }^{60}$ Esta teoría de la "suspensión del juicio" aparece formulada ya en escritos de 1680-1684. En ella, sin embargo, Leibniz aclara que la elección siempre caerá sobre lo que se juzgue mejor. Cf. A VI, iv, 1456-1457, 1445. Esta doctrina permite postular la posibilidad de corregir nuestra voluntad presente, suspendiéndola hasta una evaluación intelectual ulterior, que nos permita reorientar el deseo de un bien aparente hacia un bien real. Cf. G VI, 390-392. ${ }^{61}$ Cf. G VI, 316, 321.
}

Manuscrito - Rev. Int. Fil., Campinas, v. 38, n.1, pp. 129-165, jan.-jun. 2015. 
bondad ("el apetito de lo bueno") es la perfección de la voluntad. ${ }^{62}$ De ello se sigue que la bondad divina no puede admitir imperfección alguna, y ha de querer lo óptimo como consecuencia necesaria de la naturaleza de Dios. Por ello, Leibniz afirma expresamente que, si Dios fuera capaz de preferir un bien menor a un bien mayor, "sería imperfecto, lo mismo que el objeto de su elección". ${ }^{63}$

Este argumento tiene una consecuencia importante, y es que $T$, en tanto referida a la elección $\left(T_{i i}\right)$, debe considerarse como absolutamente necesaria. Esto se debe a que la proposición contraria conduce a una contradicción demostrable en un número finito de pasos. Tal contradicción consiste en concebir al sujeto de todas las perfecciones como carente de una perfección (la máxima bondad). Por ello, no es casual que Leibniz llegue a afirmar que "en Dios puede ser demostrado que no puede hacer nada malo". ${ }^{64}$

Este rechazo a la posibilidad de una elección divina de lo menos perfecto tiene una consecuencia problemática, y es que deja a Leibniz atrapado en una situación de contradicción modal. En efecto, si Leibniz se refiere a $T$ como una tesis unívoca en su significación, y además la considera como una verdad contingente, se sigue que hay una proposición contingente $(T)$ cuya proposición contraria (en una de sus formulaciones admitidas y analizadas por Leibniz) no es posible. Y ello infringe el criterio modal general leibniziano, según el cual lo contingente es aquello cuyo contrario es posible, aquello que siendo verdadero podría ser falso. En términos más esquemáticos: para Leibniz

$T$ es contingente (bajo la interpretación $T_{i}$ ); pero

${ }^{62}$ Leibniz lo afirma expresamente en Causa Dei. Cf. G VI, 441.

${ }^{63}$ Cf. G VI, 387.

${ }^{64} \mathrm{Gr} 333$.

Manuscrito - Rev. Int. Fil., Campinas, v. 38, n.1, pp. 129-165, jan.-jun. 2015. 
$\neg T$ no es posible (bajo la interpretación $T_{i i}$ ).

Esta virtual contradicción surge de la ambigüedad presente en el análisis modal leibniziano de la elección divina, que oscila entre la modalización de la elección y la de su objeto. Para evitar esta consecuencia, Leibniz hubiera tenido que esclarecer y establecer una única significación para $T$ y su contraria, o bien, hubiese tenido que trazar una clara distinción entre $T_{i}$ y $T_{i i}$, permitiendo un tratamiento diferenciado. ¿Cuál de estas vías escogió?

\section{La vía de solución vislumbrada hacia 1706}

En las notas sobre Bayle, redactadas hacia 1706, Leibniz formula lo que puede considerarse su análisis más elaborado e innovador del problema modal en torno a T.65 Allí, intenta trazar la distinción entre dos sentidos de la proposición "Dios elige lo mejor". Su formulación de ambos sentidos se basa en una apropiación, no muy clara, de la distinción medieval entre proposiciones modales de dicto y de re.

Leibniz no emplea las expresiones "de dicto"/“de re", pero las formulaciones que ofrece se ajustan a la tradicional distinción, que diferenciaba un modo adverbial o de re ("A es necesariamente B") de un modo nominal o de dicto ("A es B" es necesario). ${ }^{66}$ En términos semánticos, los autores medievales asumían que las proposiciones modales de re expresaban el modo en que el predicado pertenece al sujeto, por lo cual dicho modo era atribuido a las cosas mismas (res). En

\footnotetext{
65 Cf. Gr 493-494.

${ }^{66}$ Cf. Knuuttila (2008, p. 534). Leibniz se refiere a tal distinción en términos de proposiciones en sentido compuesto y en sentido dividido, de acuerdo a la caracterización tomada por Abelardo de las Refutaciones sofísticas de Aristóteles. Cf. Gr 475
}

Manuscrito - Rev. Int. Fil., Campinas, v. 38, n.1, pp. 129-165, jan.-jun. 2015. 
las proposiciones de dicto, por el contrario, la modalidad se atribuía al significado global expresado por una proposición (dictum). ${ }^{67}$ Se asumía, también, que una proposición modalizada podía ser verdadera o falsa según se interpretara en un sentido o en otro. ${ }^{68}$

Leibniz no clarifica en qué modo comprende esta distinción. Lo que hace es afirmar que, bajo una formulación (que correspondería al modo adverbial o de re), $T$ es contingente, mientras que bajo la otra formulación (nominal o de dicto), ella es necesaria. Leibniz explica esta diferencia asociando cada versión de $T$ a una proposición que expresaría su sentido bajo esa formulación. Su análisis puede esquematizarse del siguiente modo: ${ }^{69}$

\begin{tabular}{|l|l|l|}
\hline $\begin{array}{l}\text { Modalidad } \\
\text { tradicional }\end{array}$ & $\begin{array}{l}\text { Formulación } \\
\text { leibniziana }\end{array}$ & $\begin{array}{l}\text { Equivalencia propuesta } \\
\text { por Leibniz }\end{array}$ \\
\hline de re & $\begin{array}{l}\text { Dios quiere necesariamente lo } \\
\text { mejor }\end{array}$ & $\begin{array}{l}\text { Esto (que es elegido) es } \\
\text { (necesariamente) lo mejor }\end{array}$ \\
\hline de dicto & "Dios quiere lo mejor" es & "Esto que es lo mejor es \\
& necesario & elegido" es necesario \\
\hline
\end{tabular}

En estas equivalencias propuestas por Leibniz, la formulación de re coincide con $T_{i}$ en su modalización de cuál objeto es el mejor, mientras que la formulación de dicto parece coincidir, también, con $T_{i i}$ en la modalización del modo en que Dios elige (bajo la consideración de lo mejor). Según argumenta Leibniz, la proposición "Dios quiere lo mejor" es necesaria, porque "Dios es necesariamente aquel que quiere lo

${ }^{67}$ Cf. Langerlund (2012).

${ }^{68}$ Cf. Knuuttila (2008, p. 520-521).

${ }^{69}$ Cf. Gr 493.

Manuscrito - Rev. Int. Fil., Campinas, v. 38, n.1, pp. 129-165, jan.-jun. 2015. 
mejor". ${ }^{70}$ Pero ello no implica que Dios quiera necesariamente lo óptimo, porque la proposición "esto es lo mejor" es indemostrable, lo cual remite implícitamente al criterio del análisis infinito. Ello, en definitiva, garantiza que Dios quiere lo óptimo libremente.

Este abordaje resultaba enormemente promisorio. En él, Leibniz reconoce expresamente la necesidad analizar la tesis de la elección divina distinguiendo claramente los dos sentidos que ya había reconocido en ella. Asimismo, admite que, en uno de esos sentidos, la tesis es necesaria. Lamentablemente, Leibniz no profundizó este análisis. En lugar de ello, pasó a sostener, desde 1707, que la elección divina es moralmente necesaria. Si hubiera profundizado esta vía, habría tenido que introducir y explicar una nueva distinción modal: aquella entre formulaciones nominales (de dicto) y adverbiales (de re). Pero esto era problemático, porque Leibniz siempre empleó las modalidades aléticas para cualificar proposiciones, eventos y cosas, sin introducir distinciones semánticas. Como lo muestra el quinto escrito a Clarke, su concepción modal siguió asentada, en su madurez, sobre la distinción entre una necesidad absoluta y una necesidad hipotética. A esta distinción, Leibniz solamente agregó otra: aquella entre necesidad absoluta y necesidad moral. ${ }^{71}$

Sin embargo, este análisis leibniziano permite esbozar, a modo de hipótesis de trabajo, una interpretación según la cual habría, en definitiva, una diferencia semántica ineliminable en $T$, que exige la distinción entre dos sentidos, o más bien, entre dos proposiciones diferentes (vía que Leibniz podría haber seguido para no introducir la distinción de dicto/de re). En un primer sentido, $T$ parece referir a la regla o el criterio general según el cual Dios elige, siempre que elige. Este es, a

\footnotetext{
${ }^{70}$ Cf. Gr 494.

${ }^{71}$ Cf. G VII, 389.
}

Manuscrito - Rev. Int. Fil., Campinas, v. 38, n.1, pp. 129-165, jan.-jun. 2015. 
mi juicio, el sentido más relevante de la tesis, y el que requiere una cuantificación universal. Bajo esta significación, que correspondería a $T_{i i}$, la proposición podría formularse (en forma simplificada) como "Dios elige siempre lo mejor", y sería absolutamente necesaria, ya que es demostrable a partir de la esencia divina y de la concepción leibniziana de la voluntad. En un sentido secundario, $T$ parece referir a la elección efectiva de un objeto determinado por parte de Dios, por lo cual podría formularse como "Dios eligió lo mejor" (por ejemplo, cuando eligió crear el mundo actual). Esta versión correspondería a $T_{i}$, y expresaría una verdad contingente, porque ella sí involucraría la consideración de cuál objeto es el mejor, como sugiere Leibniz, y esto requeriría de un análisis infinito.

Esta interpretación requiere un análisis más profundo, pero cuenta con apoyo textual, ya que Leibniz mismo intentó trazar una distinción similar, haciendo de ella una vía de solución a las aporías que le planteaba la elección divina de lo óptimo.

\section{Conclusiones}

Hacia 1680, Leibniz parece asumir que la compatibilidad entre una la libertad suprema y una necessitas eligendi optimum, en Dios, resultaba problemática, y comienza a abordar el desafío de clarificar el carácter modal de la proposición "Dios elige lo mejor". La posición principal que sostuvo desde entonces, aunque no sin oscilaciones, fue favorable a la contingencia de tal proposición. Ahora bien, la justificación de esta posición lo enfrentó a una dificultad modal compleja. Esta dificultad surgía del propio tratamiento leibniziano de las nociones modales.

En efecto, Leibniz siempre empleó las modalidades aléticas para cualificar, de manera indistinta, proposiciones, eventos e incluso cosas. En todos los casos, su comprensión de la contingencia implicaba la 
posibilidad de lo contrario. Sin embargo, Leibniz admite dos interpretaciones distintas de lo contrario a una elección divina de lo óptimo. Esta ambigüedad, a su vez, entraña dos interpretaciones diferentes de la tesis analizada. Bajo una de ellas, el modalizador cualifica al objeto que es elegido, de modo que la tesis resulta necesaria si y sólo si su objeto es necesario, o bien, si es necesario que sea el mejor. Según la otra interpretación, el modalizador cualifica más bien la elección, el modo en que Dios elige, por lo cual la proposición será necesaria si y sólo si es necesario que Dios elija en razón de lo mejor.

Leibniz justifica la contingencia de la tesis en base a la primera interpretación, argumentando que lo contrario a lo que Dios elige sigue siendo posible. Asimismo, rechaza expresamente la posibilidad de la proposición contraria bajo la segunda lectura. Para él, la proposición "Dios elige aquello que no es mejor" resulta imposible, ya que es contraria a su concepción de la voluntad, al principio de razón suficiente, e implica contradicción con la absoluta perfección divina. El problema es que Leibniz no diferencia las dos interpretaciones de la tesis como dos proposiciones distintas. En consecuencia, su posición conduce a una contradicción modal: hay una misma proposición que es contingente, pero que admite una interpretación bajo la cual su contraria no es posible.

Si este análisis es correcto, cabe afirmar que, en la metafísica leibniziana, la razón suficiente de la que se deriva la existencia del mundo tiene un estatuto modal ambiguo, y constituye una suerte de excepción en su teoría modal. Se trata de un principio que, para Leibniz, es contingente, pero cuyo contrario no es posible, o no lo es al menos en su significación más relevante.

El escrito de 1706, en el cual intenta clarificar la modalidad de la elección divina aplicando la distinción entre modalidades de dicto/ de re, evidencia cuán consciente era Leibniz de este problema. Allí reconoce

Manuscrito - Rev. Int. Fil., Campinas, v. 38, n.1, pp. 129-165, jan.-jun. 2015. 
expresamente que la proposición es necesaria cuando refiere a la elección (versión de dicto), pero contingente cuando refiere al objeto elegido (versión de re). Sin embargo, este análisis requiere la fundamentación de una distinción semántica entre aserciones modales nominales y adverbiales, y Leibniz nunca formuló semejante explicación.

No obstante, este intento sugiere que sería legítimo, incluso para Leibniz, distinguir dos proposiciones diferentes en el análisis modal de la tesis: una referiría al criterio general según el cual Dios elige, y otra referiría a su elección efectiva de un objeto específico. La primera sería absolutamente necesaria, mientras que la segunda sería contingente, según los propios argumentos leibnizianos. Tal distinción permitiría evadir la contradicción modal aquí señalada. Sin embargo, ella sería insuficiente para preservar la libertad divina de toda sombra de necesidad metafisica, ya que seguiría siendo una verdad demostrable que Dios elige siempre en razón de lo mejor.

La ambigüedad de la posición leibniziana expresa, según creo, la encrucijada teórica a la que lo enfrentaba la elección divina de lo óptimo. Para Leibniz, tal elección no puede ser necesaria, porque ello suprimiría la libertad de Dios y lo despojaría de toda cualidad moral. Sin embargo, una elección divina de lo menos perfecto no puede ser posible, porque ella derrumbaría un edificio metafísico que va desde el principio de razón suficiente hasta la misma teodicea, pasando por el rechazo del molinismo. Esta encrucijada revela una de las aporías más hondas que laten en el corazón de la metafísica leibniziana. Tal metafísica parece requerir la apelación a un principio híbrido en su carácter modal, que sea esencialmente contingente, para asegurar que la obra de Dios no es el fruto de una necesidad ciega y ajena a todo propósito moral, pero que participe de algún modo de la necesidad, para asegurar que la bondad divina no es el resultado azaroso de una voluntad indiferente al bien. 


\section{EDICIONES DE LOS ESCRITOS DE LEIBNIZ}

LEIBNIZ, G. W. Sämtliche Schriften und Briefe. (A) Herausgegeben von der Berlin-Branderburgischen Akademie der Wissenschaften und der Akademie der Wissenschaften in Göttingen. (Darmstadt, 1923 y sgts., Leipzig, 1938 y sgts., Berlin, 1950 y sgts., Münster, 1999 y sgts.)

- Opuscules et fragments inédits de Leibniz. Extrait des manuscrits de la Bibliothèque royale de Hanovre. (C) Paris: Félix Alcan, 1903; reimpresión Hildesheim: Georg Olms, (1961).

- Die philosophischen Schriften. (G) Ed. C. I. Gerhardt, 7 vols. Berlin, Weidman, 1875-1890; reimpresiones Hildesheim, Georg Olms, 1960-61 y Hildesheim-New York, Georg Olms, (1978).

- Testes inédits d'après les manuscrits de la bibliothèque provinciale de Hanovre. (Gr) Ed. G. Grua, 2 vols. Paris: Presses Universitaires, 1948.

\section{TRADUCCIONES AL INGLES}

LEIBNIZ, G. W. Philosophical Papers and Letters, Ed. and tr. Leroy E. Loemker. Chicago: Chicago University Press, 1956. Second editon. Dordrecht: Kluwer Academic Publishers, 1969.

SLEIGH, R. C. Jr., Papers Concerning the Problem of Evil, 1671 1678. (Sl) New Haven \& London: Yale University Press, 2001. 


\section{TRADUCCIONES AL CASTELLANO}

OLASO, E. (comp.), G. W. Leibniz. Escritos filosóficos. (de Olaso) Buenos Aires: Charcas, 1982.

LEIBNIZ, G. W. Escritos en torno a la libertad, el azar y el destino. (Roldán) Concha Roldán Panadero (comp.) Madrid: Tecnos, 1990.

Los textos de Leibniz se citarán indicando la edición y su ubicación (tomo y/o número de página). Los textos de las ediciones en castellano se citarán indicando también su ubicación en alguna de las ediciones en idioma original. Donde no esté indicado, las traducciones son mís.

\section{BIBLIOGRAFÍA SECUNDARIA}

ADAMS, R. M. "Must God Create the Best?". Philosophical Review, 81, pp. 317-332, 1972.

-_Leibniz's Theories of Contingency". Rice University Studies, 63, no 4, pp. 1-41, 1977.

—_. "Divine Necessity". Journal of Philosophy, 80, pp. 741-752, 1983. - .Leibniz. Determinist, Theist, Idealist. New York: Oxford University Press, 1994.

- . "Moral Necessity". En: RUTHERFORD, D. \& COVER, J. A. (eds.) (2005), pp. 181-193.

ANFRAY, J.P, "Leibniz, le choix du meilleur et la nécessité morale". En: RATEAU, Paul (ed.) (2011), pp. 59-78.

BEGBY, E. "Leibniz on Determinism and Divine Foreknowledge". Sutida Leibnitiana, 37, pp. 83-98, 2005. 
BLUMENFELD, D. "Leibniz`s Theory of the Striving Possibles". Studia Leibnitiana, 5, pp. 163-177, 1973.

_- "Is the Best Possible World Possible?". Philosophical Review, 84, pp. 163-177, 1975.

-. "Superessentialism, Counterparts, and Freedom". En: HOOKER (ed.) (1982), 103-123.

BROAD, C. D. "Leibniz's Predicate-in-Notion Principle and Some of its Alleged Consequences". En: FRANKFURT, H. (ed.) (1972), pp. 1-18.

BROWN, S. Leibniz. Minneapolis: University of Minnesota Press, 1984.

- The Young Leibniz and his Philosophy (1646-76). Dordrecht: Kluwer Academic Publishers, 1999.

COUTURAT, L. La logique de Leibniz. Paris: Félix Alcan, 1901. Reimpresión Hildesheim: Georg Olms (1961).

- - "On Leibniz's Metaphysics". En: FRANKFURT, H. (ed.) (1972), pp. 19-45.

CURLEY, E. M. “The Root of Contingency”. En: FRANKFURT, H. (ed.) (1972), pp. 69-97.

DAVIDSON, J. D. "Video meliora proboque, deteriora sequor. Leibniz on the Intellectual Source of Sin". En: RUTHERFORD, D. \& COVER, J. A. (eds.) (2005), pp. 234-253.

DASCAL, M. Leibniz: What Kind of Rationalist? Tel Aviv: Springer, 2008.

ESCOBAR VIRÉ, M. "El problema del continuum y sus implicancias en la teoría leibniciana de la sustancia". Revista de Filosofía y Teoría Política, 38, pp. 67-93, 2007.

- "La constitución de lo imaginario como modo de representación en Leibniz". En: PAVESI, P. (et.al) (2010), pp. 125-144.

Manuscrito - Rev. Int. Fil., Campinas, v. 38, n.1, pp. 129-165, jan.-jun. 2015. 
-_. "El argumento ontológico, la necesidad absoluta y el problema de la contingencia en Leibniz". Revista Latinoamericana de Filosofía, Vol. XXXVII, No 1, pp. 97-125, 2011.

ESQUISABEL, O. "El tratamiento de las modalidades aléticas y deónticas en Leibniz", en LEGRIS, J. (comp.) (2011), pp. 7-23.

FRANKEL, L. "From a Metaphysical Point of View : Leibniz and the Principle of Sufficient Reason". En: WOOLHOUSE, R. S. (ed.) (1994).

FRANKFURT, H. (ed.), Leibniz: A Collection of Critical Essays. Garden City, New York: Doubleday Anchor, 1972.

FRIEDMANN, G. Leibniz et Spinoza. Paris: Gallimard, 1946.

GRUA, G. Jurisprudence universelle et Théodicée selon Leibniz. Paris : Presses Universitaires de France, 1953.

HACKING, I. "Infinite Analysis". Studia Leibnitiana, 6, pp. 126-130, 1974.

—. "A Leibnizian Theory of Truth". En: HOOKER, M. (ed.) (1982), pp.185-195.

HOOKER, M. (ed.), Leibniz. Critical and Interpretive Essays. Minneapolis: University of Minnesota Press, 1982.

HOSTLER, J. "Some remarks on 'omne possibile exigit existire". Studia Leibnitiana, 5, pp. 281-285, 1973.

—_. Leibniz's Moral Philosophy. Harper \& Row Publishers, 1975.

JOLlEY, N. The Cambridge Companion to Leibniz. Cambridge: Cambridge University Press, 1995.

LEGRIS, J. Documentos del CIECE 7. Buenos Aires: Centro de Investigación en Epistemología de las Ciencias Económicas (Facultad de Ciencias Económicas, Universidad de Buenos Aires), 2011.

LOVEJOY, Arthur Onken, 1972, "Plenitude and Sufficient Reason in Leibniz and Spinoza". En: FRANKFURT, H. (ed.) (1972), pp. 281-334. 
MOLL, K. "Deus sive harmonia universalis est ultima ratio rerum: the conception of God in Leibniz's early philosophy". En: BROWN, S. (ed.) (1999), pp. 65-78.

MONDADORI, F. "Reference, Essentialism and Modality in Leibniz`s Metaphysics”, Studia Leibnitiana, 5, pp. 74-101, 1973.

- "Leibniz and the Doctrine of Inter-World Identity". Studia Leibnitiana, 7, pp. 21-75, 1975.

- - "Necessity ex Hypothesi". En: Centro Fiorentino, The Leibniz Renaissance, pp. 191-222, 1986.

MURRAY, M. "Leibniz on Divine Foreknowledge of Future Contingents and Human Freedom". Philosophy and Phenomenological Research, 55, pp. 75-108, 1995.

-. "Intellect, Will and Freedom: Leibniz and his Precursors". Leibniz Review, 6, pp. 25-59, 1996.

- "Spontaneity and Freedom in Leibniz". En: RUTHERFORD, D. \& COVER, J. A. (eds.) (2005), pp. 194-216.

PARKINSON, G. H. R. "Leibniz on Human Freedom". Wiesbaden, 1970.

- "Sufficient reason and human freedom in the Confessio Philosophi”, en BROWN, S. (ed.) (1999), pp. 199-222.

PASINI, E. "Complete Concepts as Histories". Studia Leibnitiana, 42, pp. 229-243, 2012.

PAVESI, P. (et.al) Entre pensar y sentir: estudios sobre la imaginación en la filosofía moderna. Buenos Aires: Prometeo Libros, 2010.

PIRO, F. "Leibniz and ethics: the years 1669-72". En: BROWN, S. (ed.) (1999), pp. 147-167.

- "For a History of Leibniz's Principle of Sufficient Reason. First Formulations and their Historical Background". En: DASCAL, M. (ed.) (2008), pp. 463-478.

POSER, H. Zur Theorie der Modalbegriffe bei G.W. Leibniz, Studia Leibnitiana Supplementa VI. Wiesbanden: Franz Steiner Verlag, 1969.

Manuscrito - Rev. Int. Fil., Campinas, v. 38, n.1, pp. 129-165, jan.-jun. 2015. 
RATEAU, P. La question du mal chez Leibniz. Fondaments et élaboration de la Théodicée. Paris: Honoré Champions Éditeur, 2008.

- Lectures et interprétations des Essais de théodicée de G. W. Leibniz, Studia Leibnitiana - Sonderhefte 40. Stuttgart: Franz Steiner Verlag, 2011.

RESCHER, N. "Contingence in the Philosophy of Leibniz". The Philosophical Review, 61, pp. 26-39, 1952.

- The Philosophy of Leibniz. Englewood Cliffs: Prentice-Hall, 1967.

- Leibniz's Metaphysics of Nature. Dordrecht: D. Reidel Publishing Company, 1981.

- . "Contingentia Mundi. Leibniz on the World`s Contingency". Studia Leibnitiana, 33, pp. 145-162, 2001.

-_. "Leibniz on God`s Free Will and the World`s Contingency". Studia Leibnitiana, 34, pp. 208-220, 2002.

- "On Some Purported Obstacles to Leibniz's Optimalism". Studia Leibnitiana, 37, pp. 131-146, 2005.

RICE, H., "Fatalism", The Stanford Encyclopedia of Philosophy (Fall 2009 Edition), Edward N. Zalta (ed.), URL = http://plato.stanford.edu/archives/fall2009/entries/fatalism/, 2009.

RUSSELL, B. Exposición crítica de la filosofía de Leibniz. Buenos Aires: Siglo veinte, 1977.

RUTHERFORD, D. Leibniz and the Rational Order of Nature. Cambridge: Cambridge University Press, 1995.

RUTHERFORD, D. \& COVER, J. A. Leibniz. Nature and Freedom. New York: Oxford University Press, 2005.

SAAME, O. El principio de razón en Leibniz, Barcelona: Laila, 1985.

SCHEPERS, H. "Posibilidad y contingencia. Historia de la terminología filosófica anterior a Leibniz". Revista de Filosofía y Teoría Politica, 33, pp. 43-63, 1999. 
SLEIGH, R. C. (Jr.) "Truth and Sufficient Reason in the Philosophy of Leibniz”. En: HOOKER, M. (ed.) (1982), pp. 209-42.

- Leibniz \& Arnauld. A Commentary on Their Correspondence. New Haven/London: Yale University Press, 1990.

WILSON, M. “On Leibniz`s Explication of Necessary Truth”. En: FRANKFURT, H. (ed.) (1972), pp. 401-419.

WOOLHOUSE, R. S. Gottfried Wilhelm Leibniz. Critical Assessments Vol. 1. New York: Routledge, 1994. 
